\title{
Lessons Learned: Management of Breast Cancer Patients throughout the COVID-19 Pandemic
}

\author{
Mary E. F. Ramirez ${ }^{1}$. Jill R. Dietz ${ }^{1}$
}

Accepted: 7 September 2021 / Published online: 17 November 2021

๑ The Author(s), under exclusive licence to Springer Science+Business Media, LLC, part of Springer Nature 2021

\begin{abstract}
Purpose of Review The COVID-19 pandemic of 2020 presented a multitude of challenges for physicians nationwide. Standard of care treatment was tailored to protect patients from virus exposure, while delivering safe and adequate care. This article reviews the steps taken to treat and protect breast cancer patients during the pandemic and reentry approaches to resume care. Recent Findings Breast cancer experts rapidly convened to develop treatment guidelines during the pandemic. These recommendations encompass screening approach, prioritization of breast cancer patients, educational modifications, research and data considerations, and a re-entry treatment approach as the pandemic evolved. Without prior experience with a pandemic of this magnitude, these guidelines were based on expert knowledge and previously established data.

Summary This emergency forced physicians to operate in a more efficient and effective manner to deliver value-based patient care, and future evaluation of these adjustments will determine if overall patient outcomes were compromised.
\end{abstract}

Keywords Breast cancer and COVID $\cdot$ Management during pandemic $\cdot$ Treatment guidelines $\cdot$ COVID re-entry $\cdot$ Breast cancer prioritization

\section{Introduction}

On March 13, 2020, the President of the USA declared a national emergency due to the rapid spread of the coronavirus, COVID-19. Shortly thereafter, a succession of restrictions were released across the country, including the closing of schools followed by nonessential businesses. Social distancing shortly followed with mandated stay at home orders being issued state by state in an effort to control the spread of this novel virus. Within just a few weeks, on 4/6/2020, COVID-19 deaths became the leading cause of death in the USA [1]. Concerns quickly arose regarding hospitals becoming overwhelmed with patients causing severe shortages of personal protective equipment (PPE) for front-line

This article is part of the Topical Collection on Breast Cancer Management During the COVID-19 Pandemic

Mary E. F. Ramirez

Mary.Freyvogel@uhhospitals.org

1 University Hospitals Cleveland Medical Center, St. John Medical Center, 29000 Center Ridge Rd., Westlake, OH 44145, USA healthcare workers. Due to these concerns, physicians started using telemedicine whenever possible, and surgeons were asked to cancel 'elective' surgeries to preserve PPE. This pandemic rapidly presented a multitude of challenges that physicians nationwide had to quickly adjust to with the common goal of minimizing exposure risk and preserving resources, yet continuing to provide optimal patient care. This posed a particularly unique challenge in the setting of cancer treatment, and this article will specifically address the surgical management of breast cancer during the COVID-19 pandemic.

Without prior experience with a medical emergency of this magnitude, breast cancer leaders nationwide quickly assembled to generate a multidisciplinary expert opinion and provide much needed guidance to fellow treating physicians. Representatives from the American Society of Breast Surgeons (ASBrS) and the American College of Radiology (ACR) collaborated to put forth guidelines for screening mammography during the pandemic [2]. It became clear that continuing elective screening posed a greater risk than benefit to patients; so on March 26, 2020, the ASBrS and ACR recommended medical facilities postpone all breast screening, effective 
immediately. This recommendation was to be re-evaluated on a weekly basis based upon the geographic impact of the virus.

The ASBrS forum, a large community chat room for members, quickly became overwhelmed with requests for assistance in treating breast cancer patients during the surgery shutdown. The breast cancer consortium was quickly created, comprising members from the ASBrS, National Accreditation Program for Breast Centers (NAPBC), National Comprehensive Care Network ( $\mathrm{NCCN}$ ), Commission on Cancer ( $\mathrm{CoC})$, and ACR. This expert group assembled to develop treatment recommendations based on a prioritization of breast cancer patients [3••]. Within a week, they wrote and edited these recommendations, which were then posted by all participating organizations, including the American College of Surgeons (ACS), to make them readily available to breast cancer physicians everywhere [4]. Shortly thereafter, the priority recommendations were published in a peer-reviewed journal and

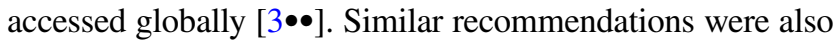
created by other university breast centers, such as Dana-Farber Cancer Institute and University of Pittsburgh Magee Women's Hospital $[5,6]$. These recommendations were not meant to supersede individual physician judgment or institutional policies/guidelines, rather, give treating physicians a guide to follow during such an uncertain time.

The impact of coronavirus on the cancer patient can be significant due to the need for multiple visits to a healthcare facility as well as the immunocompromised state caused by the disease itself and proposed treatments. These patients may be more susceptible to infection, and recent small case studies have shown worse outcomes from coronavirus in cancer patients than those without cancer [7-9]. Patients having received chemotherapy or surgery within 30 days of contracting COVID-19 carried a higher risk of severe events compared to those who had not [8]. Cancer patients are also often older with more comorbidities increasing their risk of complications [9]. Careful consideration must be made in the cancer population to both protect them from exposure and provide effective and safe treatment of their disease. Safety measures should include implementation of strict infection control and treatment in the outpatient setting rather than hospitalization, whenever safe and feasible. The following is a review of the recommended prioritization in the breast cancer population, provided by 'The COVID-19 pandemic breast cancer consortium' during this unprecedented time $[3 \bullet \bullet$.

\section{Prioritization Recommendations}

Breast cancer patients were divided into 'Priorities' based on the severity of their disease as well as the potential risks and benefits of the required treatment. These priorities were created across all disciplines of breast cancer management.
'Priority A' included patients with an immediately lifethreatening or clinically unstable conditions. These patients were assigned top priority for urgent treatment, regardless of resource availability.

'Priority B' included patients who did not have a lifethreatening condition, but whose treatment should not be indefinitely delayed. This priority captures a large majority of breast cancer patients and a short delay in treatment (6-12 weeks) was not felt to impact overall outcome. Subclassifications were created within this group to assist with further prioritization, should further delay become necessary.

'Priority C' included patients whose treatment could safely be delayed indefinitely with no negative impact on overall outcome.

These priority groups were applied to each facet of the multidisciplinary treatment of breast cancer, including initial work-up in the office, imaging studies, surgical oncology, medical oncology, and radiation oncology. We will address the workup and surgical management here.

\section{Outpatient Workup}

The expert consortium recommended that the majority of outpatient encounters be conducted via telemedicine in efforts to reduce the risk of viral transmission between patients and healthcare workers. At this virtual visit, the physician can further evaluate the potential need for an in-person encounter. Priority A patients would include anyone in need of an in-person visit, for example, unstable postoperative patients. Priority B patients should be evaluated in person by one of the multidisciplinary team members initially. This would include newly diagnosed breast cancer patients, established patients with a new breast complaint requiring further workup, and routine postoperative patients. Priority $\mathrm{C}$ patients can be seen virtually or delayed until after the pandemic. This category would include routine follow-up, survivorship visits, or high-risk screening.

\section{Breast Imaging}

Patients may be brought in for breast-specific imaging if designated Priority A. These patients may have urgent issues such as breast abscess or serious postoperative complication. Priority B patients would include diagnostic imaging for an abnormal screening study, biopsies for a BIRADS-4 or 5 lesion, breast MRI for further evaluation of newly diagnosed breast cancer or to assess response to chemotherapy. Lower suspicion lesions such as BIRADS-4a could be postponed, and BIRADS-3 patients due for follow-up imaging could be delayed until after the pandemic. All screening studies could be placed in Priority $\mathrm{C}$ and postponed until after the pandemic. 


\section{Surgical Oncology}

The COVID-19 breast cancer consortium formulated a list of patient scenarios by priority in regard to surgical management of their disease. The overall objective in delaying surgeries is to preserve the necessary PPE required to manage the expected influx of COVID-19 patients. In order to avoid adversely impacting breast cancer outcomes, multidisciplinary input is recommended in the decision-making process of which patients' treatments can be safely delayed.

Priority A patients include those requiring immediate surgical attention such as abscess drainage or expanding postoperative hematoma. Priority B patients include high-risk cancer patients with more aggressive tumor biology, such as triple negative and HER2+. Patients completing neoadjuvant chemotherapy should also be kept on a strict schedule for surgery as they would be at risk for disease progression if delayed. Patients with hormone-sensitive cancers can be considered for neoadjuvant endocrine therapy to temporize their disease until surgery is more feasible. Finally, Priority $C$ patients would include earlystage disease such as ductal carcinoma in situ (DCIS) and clinically node-negative, hormone-positive invasive disease. These cases can be delayed, and/or hormonal therapy can be initiated for temporization. High-risk lesions, benign lesions, and prophylactic surgeries are recommended to be delayed until after the pandemic.

Similar recommendations arose from highly respected institutions, such as Dana-Farber Cancer Institute and Magee Women's Hospital (UPMC) [5, 6]. There was a general similarity among the consensus recommendations. Dana Farber recommended clinical monitoring every 8 weeks to evaluate for disease progression [5]. UPMC recommendations also mirrored the consortiums with the common goals of preserving PPE, limiting in-person visits and delaying non-urgent surgical procedures. The risk/benefit ratio of delaying surgery should be considered for each patient individually. If the risk of postponing breast cancer surgery poses little to no harm, this should be a consideration and discussed with the patient [6].

With the assistance and guidance from these breast cancer experts, physicians were quickly tailoring their practice to accommodate both the pandemic risks and necessity for patient care. Several areas of overlap are seen among these recommendations, and multidisciplinary input is ideal and recommended for all patients. The goal of these recommendations is to help guide the decision-making process for breast cancer patients by weighing the risk that cancer treatment may increase the susceptibility of COVID-19 infection vs the risk of adverse oncologic outcomes from delaying their treatment until the pandemic resolved.

\section{Educational Modifications}

In addition to surgeries and in-person visits being tailored, national educational conferences in 2020 were widely canceled due to travel restrictions and the need for social distancing.
These conferences are largely relied upon by physicians nationwide for ongoing education, and there was a call to action to continue to provide relevant educational opportunities. One such conference by the ASBrS was quickly adjusted to accommodate physicians from a distance. The leadership committee transformed this largely attended in-person meeting to a virtual one. The society was already offering weekly virtual breast fellow education, which was then expanded to the entire breast community. Multidisciplinary COVID-19 tumor boards were broadcasted and many of the consortium members participated. The newly created virtual 2020 meeting series delivered topics to assist with the treatment of breast cancer patients during the pandemic. Some relevant lecture topics included 'Endocrine therapy', 'Pain management and Enhanced Recovery After Surgery (ERAS) procedures for same day mastectomy' and 'Coding tips on billing for telemedicine', to name a few. In addition to the ASBrS, several societies strengthened their year-round virtual educational platform in order to contribute to the mission of continuing to educate treating physicians. The common goals of these virtual meetings are to provide treatment recommendations, discuss changes to coding and reimbursement, offer data collection opportunities, and highlight legislative and regulatory updates. Unique 'hands-on' virtual teaching models are also being evaluated to provide small group learning and certification for oncoplastic, ultrasound, and stereotactic techniques. The ASBrS created a COVID-19 Resource Center that includes published recommendations regarding surgery, treatment, and screening during the pandemic. Quick links are provided to the top published papers as well as information for billing and coding in the newfound setting of telehealth visits. (https:// www.breastsurgeons.org).

Another focus has been providing a platform for members and industry partners to interact and stay updated on the latest technology. Industry partners have sponsored virtual webinars, and exhibit halls have been well attended, both of which have served to enrich our educational platform. The Corporate Relations Committee and Board has shifted in-person meetings with the Industry Relations Council (IRC) to virtual discussions with the shared goal of finding value in the incorporation of technology, genomic testing, and improved drugs for the treatment of breast cancer.

\section{Research and Data Collection}

Many breast cancer trials were closed to accrual early on in the pandemic with resource teams refocused on registries and studies related to COVID-19. Even though many studies have reopened, patients remain reluctant to make additional visits or contacts, and accrual numbers have not returned to normal. Dozens of manuscripts have been published in the last six months outlining care, describing 
changes in practice and looking at early outcomes of breast cancer patients during the COVID-19 pandemic. The NAPBC, ASBrS, and many other groups realized the importance of collecting data regarding the changes in practice, mandated by the pandemic. The NAPBC and ASBrS sent out a survey to members asking about practice changes and impacts of the pandemic on physicians. The ASBrS created a COVID-19-specific patient registry giving member physicians an opportunity to input patient data via a HIPAA-compliant program and see how others are treating patients during the pandemic. This was added to the existing 'Mastery' registry and to date, 234 physicians have entered data with regard to the impact of COVID-19 on 2224 patients. This will provide much needed information for ongoing research and interpretation of how patients fared during the pandemic, given the need to tailor standard of care treatment. (https://www. breastsurgeons.org/resources/covid19_registry).

With the same future goals in sight, additional organizations created similar registries of their own. The American Society of Clinical Oncology (ASCO) launched the 'ASCO Survey on COVID-19 in Oncology Registry' on April 10, 2020 [10]. This registry is meant to capture information about symptoms and severity of the virus in cancer patients as well as how the virus has impacted their care and outcomes. It aims to collect baseline and followup data during and after the pandemic in hopes of elucidating longer-term effects of COVID-19 and its impact on cancer care [10]. Access to this registry can be found at https://www.asco.org/asco-coronavirus-information/coron avirus-registry. In addition, the ACS developed a registry to collect data on COVID-19 patients who did and did not undergo surgery. This registry will capture a multitude of patients, with cancer patients comprising a subset of them [11]. All hospitals are encouraged to participate and additional information can be found at https://www.facs. org/quality-programs/covid19-registry.

\section{Re-entry Approach}

As the pandemic seemed to improve with social distancing and stay at home orders, the idea of reopening was considered. The reopening strategy would differ across the country based on geographic conditions related to PPE, COVID-19 case load, and the ability to safely accommodate high-risk patients. Similar to the prioritization recommendations, the COVID-19 pandemic breast cancer consortium again convened to publish further recommendations regarding how to safely resume care of breast

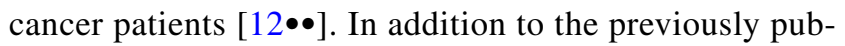
lished consortium, members from ASCO and the Society of Surgical Oncology (SSO) joined to assist with the reentry guidelines.

Breast cancer is the most common cancer among women, and restoration of care is vital as the pandemic subsides. This group of nationwide breast cancer experts developed multiple vignettes to help demonstrate the risks and benefits of reinstituting treatment. These recommendations are aimed at safely resuming multidisciplinary breast cancer care to patients whose treatment had been placed on hold or modified during the pandemic. Five primary clinical questions were identified and addressed in this manuscript and will be summarized here. The full article can be found at https://www.breastsurgeons.org/docs/covid 19/reentry.pdf?v1.

1. How do we care for our asymptomatic but high-risk patients presenting for office visits in the post-COVID era?

Breast cancer patients will begin to return to outpatient clinics and offices; however, it will be important to maintain social distancing practices to minimize close contact in waiting rooms and exam rooms. Telehealth visits are still encouraged when clinically appropriate, and physicians will need to determine who would require an in-person visit for adequate evaluation. Local infection control policies will need to be followed based on geographic region. This may include screening for symptoms or potential exposure prior to and upon arrival to the facilities. Access to routine imaging will likely be dependent on regional availability and patients should be prioritized based on screening vs diagnostic necessity. Screening vs diagnostic necessity should follow standard guidelines, and high-risk individuals requiring mammography and MRI should be prioritized when scheduling resumes. Patients requiring image-guided procedures should be screened and tested for COVID19 accordingly. Patients who test positive for COVID-19 should have their procedure delayed to protect staff from exposure.

2. How do we handle the backlog of patients whose surgical treatment was delayed due to the pandemic?

As operating rooms gradually reopen, a multitude of specialties will be contending for OR time and availability for their patients. Prioritization models should be used to help assess the urgency of cases to be rescheduled. In regard to breast cancer patients, the previously defined prioritization of patients should be used to assist with this rescheduling process. Other considera- 
tions should also include overall patient health, risk of COVID-19 exposure, and local hospital resource availability $[12 \bullet \bullet, 13]$. The consortium recommendations assign patients receiving neoadjuvant chemotherapy as high-priority patients. Conversely, those with early-stage hormone-sensitive cancers, atypia, or benign lesions will fall lower on the list of priority during the reopening phase. Massachusetts General Hospital (MGH) also published re-entry guidelines for breast cancer patients who were subject to treatment delay [14•]. Many patients managed with neoadjuvant endocrine therapy or chemotherapy will become high priority for surgery as their initial treatment comes to an end. Further delay in these patients could compromise overall outcome. To address the need for a fair and transparent system of reprioritization of surgical patients, MGH created a scoring system for surgical assignment. Aspects considered were related to risk of delaying surgery and were assigned a score by a multidisciplinary breast team. A multitude of factors were recorded for each patient including biologic details of the cancer diagnosis, time from diagnosis, completion date of neoadjuvant therapy and evidence of progression on follow-up. Records were maintained in REDCap and Microsoft Excel formats. Higher scores were deemed to carry a greater risk from delaying surgery. Scores were assigned using published data and multidisciplinary expertise. Overall scores were divided into 'very urgent', (score $\geq 30$ ), 'limited delay acceptable' (score 10-29), and 'lowest priority' (score $<10$ ). Very urgent cases have a narrow window for surgery between 2 and 4 weeks. Limited delay patients may become urgent, and surgery would ideally occur between 2 and 4 months. Lowest priority patients can safely wait until after the pandemic without anticipated harm. This tool offers a risk-stratified list of breast conditions where surgical delay was mandated during the COVID-19 pandemic. The scoring system provides a priority ranking, consistent with the consortium's recommendations, to guide practicing physicians in the care of their patients. Use of tools such as this can also provide necessary data for potential future situations where surgery may need to be delayed [14•]. This tool can be accessed at https://www.massgeneral.org/surgical-oncology/ about/news-and-events/re-entry-tool-for-breastsurgeons/.
3. As our operating rooms reopen, how should patients who were placed on endocrine therapy prior to definitive surgery be managed?

Typically, patients with early-stage, hormonesensitive breast cancers receive surgery and radiation, followed by endocrine therapy. Due to implications from the pandemic, many have been placed on endocrine therapy prior to other therapies in order to delay surgery but not effect overall outcome. Patient factors should be taken into account when reprioritizing these patients for surgery. Factors such as age, comorbidities, risk of COVID-19 infection, response to systemic therapy, and geographic resource availability are a few examples. If the patient does not show any signs of progression on endocrine therapy, either clinically or radiographically, this may be continued with plans for further repeat imaging prior to surgery. In patients receiving neoadjuvant endocrine therapy who are rescheduled for breast-conserving surgery, it is felt reasonable by the consortium to apply ACOSOG Z0011 criteria and perform sentinel lymph node biopsy alone. This is controversial and individual physician and patient comfort level is important to consider. For patients who were not initially eligible for breast conservation, 6 months of preoperative endocrine therapy could be considered to increase the potential of downsizing the tumor and converting to breast conservation. As long as the disease appears stable and continues to respond, endocrine therapy may be continued to maximize treatment effect. Repeating hormone receptors after 6-12 months of standard of care, neoadjuvant endocrine therapy is recommended. With these recommendations in place, a study published in the Journal of the American College of Surgeons established that a longer time from diagnosis to surgical treatment in this patient population did not lower overall survival [15]. To further elucidate the impact of surgical delay during the pandemic, this study evaluated 378,839 patients from the National Cancer Database (NCD) with DCIS or early-stage ER+ breast cancer treated between 2010 and 2016. Time to operation was recorded along with factors associated with pathologic upstaging. Upon final analysis, this study concluded that patients treated with neoadjuvant endocrine therapy exhibited no survival difference in regard to time to operation. Among patients receiving primary surgical treatment, surgical delays of more than 60 days were associated with pathologic upstaging in patients 
with DCIS but not invasive disease [15]. Although the patient population is not equivalent to those delayed during the pandemic, these results can provide some reassurance to treating physicians in regard to surgical delay in this patient population.

4. As we emerge from the pandemic, how do we manage patients who have already begun neoadjuvant chemotherapy?

When evaluating surgical options for a patient receiving neoadjuvant chemotherapy, consideration of current extent of the pandemic and available local resources must first be gauged. Factors intrinsic to the breast cancer diagnosis are then evaluated including subtype, treatment response, patient comorbidities, and complications during treatment. The consortium agreed that patients showing signs of progression on chemotherapy, or those with no effective alternative therapy options are considered high priority for surgery and should not have further delay [3••]. The overall approach to patients receiving neoadjuvant chemotherapy should concentrate on minimizing exposure in clinic and minimizing immunosuppression from chemotherapy. Upon completion of chemotherapy, these patients are high priority for surgery and should be scheduled. If barriers exist to scheduling surgery and the patient has the option of endocrine therapy, this can be initiated to avoid further treatment delay, however, this is not standard of care. In this extenuating circumstance, careful clinical follow-up is recommended to assess for potential progression of disease. There is limited data on outcomes of patients with cancer who contract COVID-19. Available data exhibit higher rates of complications and mortality compared to the noncancer population; however, this data was on small subsets of patients and largely focused on lung cancer patients, not breast cancer patients [7]. A study from Columbia University showed encouraging results among breast cancer patients specifically who contracted COVID-19 [16]. It was a small study of 27 breast cancer patients who were treated for coronavirus and also received breast cancer treatment within the previous 6 months. Ninety-six percent of patients were symptomatic from COVID19 and 26\% (7) were hospitalized. All patients were discharged home; however, following discharge, the only male patient died [16]. Although these results are promising, the overall aim should still concentrate on reducing clinic visits and potential exposure during treatment.
5. How do we manage a patient who is not a candidate for breast-conserving surgery but is ready for their operation?

The extent of breast surgery required determines the need for postoperative care, and during the pandemic, limiting exposure to the inpatient setting is an important factor to consider. For patients requiring mastectomy, reconstruction can still be considered, but should be limited to the affected breast and should include placement of tissue expander or implant. Autologous reconstruction is not recommended and should not be offered until conditions and resources allow. Consideration of bilateral mastectomy may be given for patients at high risk of developing contralateral breast cancer (such as young age, genetic predisposition, ER-negative disease), if local conditions allow. Decreasing length of hospital stay and complications after mastectomy, with or without reconstruction, must be carefully considered. Enhanced Recovery After Surgery (ERAS) is an approach that can be useful in this population of patients to enable patients to recover more quickly and minimize inpatient exposure $[17,18]$. The ASBrS formed an expert committee with nominated members from the ACS, SSO, American Society of Plastic Surgeons (ASPS), and The American Society of Anesthesiologists (ASA). This committee developed treatment recommendations for decreased opioid use in postoperative breast surgery patients. The manuscript also provides strategies for same day discharge for mastectomy patients which is helpful to decrease potential virus exposure [18].

\section{Conclusion}

As the pandemic continues to evolve, regional differences exist regarding COVID-19 case load and hospital resource availability which will lead to variation in the ability of institutions to reopen patient services. Primary principles for re-entry should include providing standard of care treatment while weighing risks and benefits of proposed treatments against virus exposure. Multidisciplinary input is paramount when making these treatment decisions and these recommendations should be discussed with the patient, soliciting their input while informing them of risks of delayed treatment versus risk of exposure to COVID-19. These extraordinary times have presented physicians and patients with unique challenges, and the medical community must continue to come together to confront them in the safest, most effective manner possible. The global 
collaborative efforts, multidisciplinary and multisociety partnerships, and industry engagement showcase a medical community poised to work together to protect our patients and treatment teams.

The COVID-19 pandemic of 2020 has altered the approach to patient care in a way we have never seen before. Physicians everywhere have adjusted to telemedicine and virtual visits to protect our patients from exposure while striving to deliver adequate and safe care. These modifications have been made, keeping our patients' safety and health as the top priority, and only time will tell if our adjustments have compromised overall outcomes. Major themes we have witnessed during the pandemic have increased utilization of genomic or personalized needs assessment for chemotherapy with the result of more judicious use, simplified surgeries, fewer visits and tests, and reduced number of radiation fractions for treating breast cancer patients. It is likely that the pandemic has forced us all to provide more efficient and effective medical care, decrease waste and unnecessary procedures, and find ways to engage with our patients in this new, virtual world. This unanticipated medical emergency has pushed us one step closer to an enhanced, value-based approach to patient care.

\section{Declarations}

Conflict of Interest Mary Freyvogel Ramirez and Jill Dietz declare that they have no conflict of interest.

Human and Animal Rights This article does not contain any studies with human or animal subjects performed by any of the authors.

\section{References}

Papers of particular interest, published recently, have been highlighted as:

- Of importance

$\bullet$ Of major importance

1. Goodman R, Schulkin D. Timeline of the Coronavirus Pandemic and US Response. 5/7/2020. https://www.justsecurity.org/69650/ timeline-of-the-coronavirus-pandemic-and-u-s-response/.

2. ASBrS and ACR Joint Statement on Breast Screening Exams During the COVID-19 Pandemic. March 26, 2020. https://www. breastsurgeons.org/news/?id=45.

3.• Dietz J, Moran M, Isakoff S, Kurtzman S, Willey S, Burstein H, et al. Recommendations for prioritization, treatment and triage of breast cancer patients during the COVID-19 pandemic. The COVID-19 pandemic breast cancer consortium. Breast Cancer
Res Treat 2020 Jun; 181(3)487-497. https://doi.org/10.1007/ s10549-020-05644-z. This article is from a breast cancer expert panel and was published shortly after elective surgeries were placed on hold during the COVID-19 pandemic. It provides treating physicians a prioritization of breast cancer patients to help guide decision making regarding treatment options if primary surgery was not an option. It also covers protective measures for patients and staff when instituting care.

4. COVID-19 Guidelines for Triage of Breast Cancer Patients. 3/24/2020. https://www.facs.org/covid-19/clinical-guidance/ elective-case/breast-cancer.

5. Suggested Treatment Modifications in Multidisciplinary Breast Cancer Management in the Setting of COVID-19. 3/30/2020. https://www.dana-farber.org/covidmd.

6. Soran A, Gimbel M, Diego E. Breast cancer diagnosis, treatment and follow-up during COVID-19 pandemic. Eur J Breast Health. 2020;16(2):86-8. https://doi.org/10.5152/ejbh.2020.240320.

7. Al-Shamsi H, Alhazzani W, Alhuraaui A, Coomes E, Chemaly $\mathrm{R}$, Almuhanna M, et al. A Practical approach to the management of cancer patients during the novel coronavirus disease 2019 (COVID-19) pandemic: an international collaborative group. Oncologist. 2020;25:1-10. https://doi.org/10.1634/theoncolog ist.2020-0213.

8. Al-Quteimat OM, Amer AM. The impact of the COVID-19 pandemic on cancer patients. Am J Clin Oncol. 2020;43(6):452-5. https://doi.org/10.1097/COC.0000000000000712.

9. Kuderer N, Choueiri T, Shah D, Shyr Y, Rubinstein S, Rivera D, et al. Clinical impact of Covid-19 on patients with Cancer (CCC19): a cohort study. Lancet. 2020;395(10241):1907-18. https://doi.org/10.1016/S0140-6736(20)31187-9.

10. ASCO Surgey on COVID-19 in Oncology (ASCO) Registry. April 10, 2020. https://www.asco.org/asco-coronavirus-infor mation/coronavirus-registry.

11. Collecting Important Clinical Patient Data: ACS COVID-19 Registry. A Quality Program of the American College of Surgeons. https://www.facs.org/quality-programs/covid19-registry.

12. $\bullet$ Dietz J et al. COVID-19 Pandemic Breast Cancer Consortium's Considerations for Re-entry. Breast Cancer Res Treat. 2020. This article provides a re-entry approach with multiple vignettes to demonstrate risks and benefits of resuming treatment in breast cancer patients whose treatment had been placed on hold or modified during the pandemic.

13. Prachand VS, Milner R, Angelos P, Posner M, Fung J, Agrawal $\mathrm{N}$, et al. Medically necessary, time-sensitive procedures: scoring system to ethically and efficiently manage resource scarcity and provider risk during the COVID-19 pandemic. J Am Coll Surg. 2020. https://doi.org/10.1016/j.jamcollsurg.2020.04.011.

14. Smith BL, Nguyen A, Korotkin J, Kelly B, Specht M, Spring L, et al. A system for risk stratification and prioritization of breast cancer surgeries delayed by the COVID-19 pandemic: preparing for re-entry. Breast Cancer Res Treat. 2020 Jul 25: 1-10. https://doi.org/10.1007/s10549-020-05792-2. This reference provides a tool with a risk-stratified list of breast conditions where surgical delay was mandated during the COVID-19 pandemic. The scoring system gives a priority ranking to guide practicing physicians in the care of their breast cancer patients during the reopening phase of the pandemic. 
15. Minami C, Kantor O, Weiss A, Nakhils F, King T, Mittendorf E, et al. Association between time to operation and pathologic stage in ductal carcinoma in situ and early-stage hormone receptorpositive breast cancer. J Am Coll Surg. 2020. https://doi.org/10. 1016/j.jamcollsurg.2020.06.021.

16. Kalinsky K, Accordino M, Hosi K, Hawley J, Trivedl M, Crew K, et al. Characteristics and outcomes of patients with breast cancer diagnosed with SARS-Cov-2 infection at an academic center in New York City. Breast Cancer Res Treat. 2020;182(1):239-42. https://doi.org/10.1001/jama.2020.5394.

17. Offodile AC 2nd, Gu C, Boukovalas S, Coroneos C, Chatterjee A, Largo R, et al. Enhanced recover after surgery (ERAS) pathways in breast reconstruction: systematic review and meta-analysis of the literature. Breast Cancer Res Treat. 2019;173:65-77. https://doi.org/10.1007/s10549-018-4991-8.

18. Brenin D, Dietz J, Baima J, Cheng G, Froman J, Laronga C, et al. Pain management in breast surgery: recommendations of a multidisciplinary expert panel - the american society of breast surgeons. Ann Surg Oncol. 2020. https://doi.org/10.1245/ s10434-020-08892-s.

Publisher's Note Springer Nature remains neutral with regard to jurisdictional claims in published maps and institutional affiliations. 